\title{
Giant magnon spin conductivity approaching the two-dimensional transport regime in ultrathin yttrium iron garnet films
}

\section{Xiangyang Wei ( $\nabla$ x.wei@rug.nl )}

University of Groningen https://orcid.org/0000-0002-1651-4714

Obed Alves Santos

University of Groningen

Cristhian Humberto Sumba Lusero

University of Groningen

\section{Gerrit Bauer}

University of Groningen

Jamal Ben Youssef

LabSTICC-UMR 6285

\section{Bart van Wees}

University of Groningen

\section{Letter}

Keywords:

Posted Date: January 6th, 2022

DOI: https://doi.org/10.21203/rs.3.rs-1217468/v1

License: (9) This work is licensed under a Creative Commons Attribution 4.0 International License.

Read Full License

Version of Record: A version of this preprint was published at Nature Materials on September 22nd, 2022. See the published version at https://doi.org/10.1038/s41563-022-01369-0. 


\section{Giant magnon spin conductivity approaching the two- dimensional transport regime in ultrathin yttrium iron gar- net films}

X-Y. Wei ${ }^{1, *}$, O. Alves Santos ${ }^{1}$, C.H. Sumba Lusero ${ }^{1,}{ }^{\dagger}$, G. E. W. Bauer ${ }^{1,2}$, J. Ben Youssef $^{3}$, and B.

J. van Wees ${ }^{1}$

${ }^{1}$ Physics of Nanodevices, Zernike Institute for Advanced Materials, University of Groningen, Nijenborgh 4, 9747 AG Groningen, The Netherlands

${ }^{2}$ WPI-AIMR \& Institute for Materials Research \& CSRN, Tohoku University, Sendai 980-8577, Japan

${ }^{3}$ Lab-STICC, CNRS- UMR 6285, Université de Bretagne Occidentale, 6 Avenue Le Gorgeu, 29238 Brest Cedex 3, France

*e-mail: x.wei@rug.nl

${ }^{\dagger}$ Current address: Leibniz Institute for Solid State and Materials Research, IFW, 01069 Dresden, Germany

Conductivities are key material parameters that govern various types of transport (electronic charge, spin, heat etc.) driven by thermodynamic forces. Magnons, the elementary excitations of the magnetic order, flow under the gradient of a magnon chemical potential ${ }^{1 / 3}$ in proportion to a magnon (spin) conductivity $\sigma_{m}$. The magnetic insulator yttrium iron garnet 5 (YIG) is the material of choice for efficient magnon spin transport. Here we report an unex6 pected giant $\sigma_{m}$ in record-thin YIG films with thicknesses down to $3.7 \mathrm{~nm}$ when the number of occupied two-dimensional (2D) subbands is reduced from a large number to a few, which 
corresponds to a transition from 3D to 2D magnon transport. We extract a 2D spin conductivity $(\approx 1 \mathrm{~S})$ at room temperature, comparable to the (electronic) spin conductivity of the high-mobility two-dimensional electron gas in GaAs quantum wells at millikelvin temperatures $^{4}$. Such high conductivities offer unique opportunities to develop low-dissipation magnon-based spintronic devices.

The spin current density in metals is the difference of the up- and down-spin charge current densities measured in $\mathrm{A} / \mathrm{m}^{2}$, which is driven by a gradient of the spin chemical potential (often called spin accumulation) $\nabla \mu_{s}$. The spin conductivity $\sigma$, defined as $j_{s}=j_{\uparrow}-j_{\downarrow}=\sigma_{s} \nabla \mu_{s} / e$, can be expressed in electrical units as $\mathrm{S} / \mathrm{m}$. In a magnetic insulator where charge currents are absent, each magnon carries angular momentum $\hbar$, which is equivalent to the spin current in metals carried by a pair of spin-up $(+\hbar / 2)$ and spin-down $(-\hbar / 2)$ electrons that flow in opposite directions. A magnon current $j_{m}$ can be defined as its number current times electron charge $e$. In magnetic insulator-based spintronic devices, magnon spin currents are injected, detected, and modulated by microwave striplines or electric contacts made from a heavy metal for charge-spin conversion ${ }^{5+9}$. The corresponding spin conductivity, magnon conductivity $\sigma_{m}$, is the current density divided by the gradient of the magnon chemical potential. The unit of the magnon conductivity in $j_{m}=$ $\sigma_{m} \nabla \mu_{m} / e$, where $\mu_{m}$ is the magnon chemical potential, is then the same as that of electrons in a metal ${ }^{1}$. The value of $\sigma_{m}=4 \times 10^{5} \mathrm{~S} / \mathrm{m}$ in a $210 \mathrm{~nm}$ thick YIG film at room temperature ${ }^{\sqrt{6}}$ corresponds to the electronic conductivity of bad metals.

The high magnetic and acoustic quality of magnetic insulators make them the ideal material 
for all-magnon logical circuits and magnon-based quantum information $\frac{10,11}{}$. An example of recent progress in magnon-based computing is an integrated magnonic half-adder based on $350 \mathrm{~nm}$ wide wave guides make from $85-\mathrm{nm}$-thick YIG films ${ }^{12}$. However, these devices operate with coherent magnons $(\sim \mathrm{GHz})$ excited by narrow microwave striplines which can not be integrated into an allelectrical circuit. Therefore, it is attractive to inject magnons electrically ${ }^{13}$, but those are mainly thermal $(\sim$ Thz) and scatter much stronger at phonons. Also, scalability to smaller structure sizes, essential for future high-performance processing units, requires micro and nanofabrication in all dimensions. The first step is the growth of films of a few or even a single unit cell. Previously, magnon transport was reported in transistor structures on films down to about $10 \mathrm{~nm}$, which shows that ultrathin films can maintain high quality and display intriguing non-linear magnon effects $\frac{14-15}{}$. However, the scattering by surface roughness is expected to be increase in even thinner films ${ }^{16}$. This could be an obstacle for magnon spin transport in ultrathin YIG films that hinders observation of a transition from three dimensional magnons to two dimensional magnon gas when the thermal wavelength $\lambda_{\text {thermal }}=\sqrt{4 \pi J_{s} /\left(k_{B} T\right)}\left(\sim 2 \mathrm{~nm}\right.$ at room temperature $\left.{ }^{17}\right)$ approaches the thickness of the films $t_{\mathrm{YIG}}$, where $J_{s}$ is spin-wave stiffness and $k_{B}$ is Boltzmann constant.

Here we report measurements of the magnon conductivity of YIG films with thicknesses down to $3.7 \mathrm{~nm}$. Much to our surprise, the magnon transport turns out to be strongly enhanced in the ultrathin regime. We report a drastical increase in magnon conductivity of up to $\sigma_{m}=$ $1.6 \times 10^{8} \mathrm{~S} / \mathrm{m}$ at room temperature that even exceeds the electronic spin conductivity of highpurity copper. This increase is intimately connected to the small number of occupied subbands and apparent domination by the lowest subband in our films. These results can importantly boost the 
performance of magnon-based information technology $10 \mid 18$.

We employ a non-local configuration ${ }^{6}$ (Figure 11a) of two Pt thin film strips with length $L$ at a distance $d$ on top of YIG films grown on gallium gadolinium garnet (GGG) by liquid phase epitaxy. An electric charge current $I$ through the injector generates a transverse spin current due to the spin Hall effect ${ }^{19}$, resulting in a spin accumulation $\mu_{s}$ in Pt at the interface to YIG. The injector-conversion coefficient $\eta_{\mathrm{inj}}=\mu_{s} /(e I)$ depends on the properties and dimensions of the $\mathrm{Pt}$ strip as explained in the Section I of the supplementary information (SI Section I). The effective interface spin conductance results from the exchange interaction across the interface and produces a magnon chemical potential $\mu_{m}$ on the YIG side of the interface that acts as a magnon source, where $\mu_{m} \approx \mu_{s}$ since the interface spin resistance can be ignored (see SI Section III). The detector electrode is a magnon drain that absorbs magnons and converts them into a spin current $j_{s}$ entering the Pt detector electrode. The inverse spin Hall effect generates a detector voltage $V_{\mathrm{nl}}$ with detectorconversion coefficient $\eta_{\text {det }}=V_{\mathrm{nl}} / j_{s}^{\text {det }}$. By reciprocity, $\eta_{\mathrm{inj}}=\eta_{\text {det }}$ when injector and detector contacts have the same properties (see SI Section I for details). Since the signal scales with $L$, a normalized non-local resistance can be defined as $R_{\mathrm{nl}}=V_{\mathrm{nl}} /(I L)$. The magnon conductance follows from the measured non-local resistance

$$
G_{m}=\frac{1}{\eta_{\mathrm{inj}} \eta_{\mathrm{det}}} \frac{V_{\mathrm{nl}}}{I}=\frac{R_{\mathrm{nl}} L}{\eta_{\mathrm{inj}} \eta_{\mathrm{det}}} .
$$

The magnon conductivity $\sigma_{m}$ as a function of the thickness $t_{\mathrm{YIG}}$ of the YIG films in Figure 1 then follows from the magnon spin conductance

$$
\sigma_{\mathrm{m}}=\frac{G_{\mathrm{m}} d}{t_{\mathrm{YIG}} L} .
$$


For the films with $t_{\mathrm{YIG}}$ much smaller than the magnon relaxation length $\lambda_{m}$ as well as the lateral device dimension, $\mu_{m}$ can be considered constant in the $z$ direction. Therefore, we use following equation to describe magnon diffusion $[20$

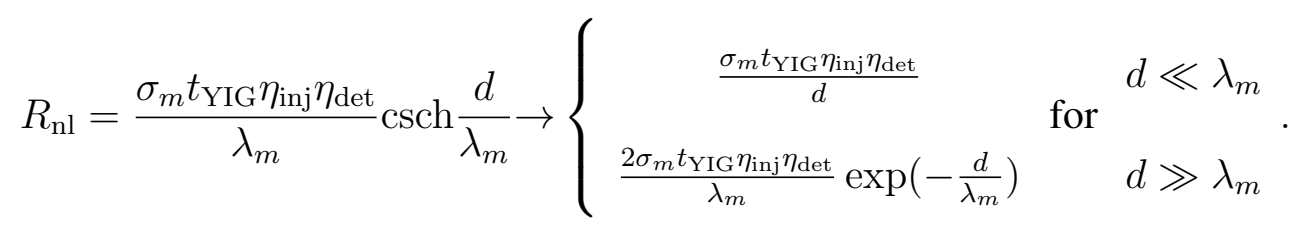

When the spacing $d$ is smaller than $\lambda_{m}$, it is the Ohmic regime in which the magnons are conserved, $R_{\mathrm{nl}}\left(G_{m}\right) \sim d^{-1}$. Otherwise, the signal decays exponentially as a function of distance due to magnon relaxation.

We measure $R_{\mathrm{nl}}$ at room temperature as a function of an external in-plane magnetic field $\mathbf{H}_{\mathrm{ex}}$ with $\left|\mathbf{H}_{\mathrm{ex}}\right|=50 \mathrm{mT}$, which we rotate in the plane (Figure 19). We modulate the AC current $I$ by a low frequency $(18 \mathrm{~Hz})$ and detect the first/second harmonic signal $V_{\mathrm{nl}}(\omega) / V_{\mathrm{nl}}(2 \omega)$ by lockin amplifiers (see Methods). $V_{\mathrm{nl}}(2 \omega)$ depends on the spin Seebeck generation and diffusion of magnons under an inhomogeneous temperature profile, which renders interpretation difficul ${ }^{21 \mid 22}$ (see SI Section V). Therefore, we focus on $V_{\mathrm{nl}}(\omega)$ that follows the formula

$$
R_{\mathrm{nl}}^{1 \omega}(\alpha)=R_{\mathrm{nl}}^{1 \omega} \cos ^{2} \alpha+R_{0}^{1 \omega},
$$

where $R_{0}^{1 \omega}$ is an offset resistance (see Methods) and $\alpha$ is the angle of $\mathbf{H}_{\text {ex }}$ with the $x$-axis. In Figure. 2, the the angle-dependent measurements in various thickness YIG films show that $R_{\mathrm{nl}}^{1 \omega}$ becomes four times larger when the film is over fifty times thinner from $210 \mathrm{~nm}$ to $3.7 \mathrm{~nm}$. We also observe a strongly increased non-local signal in ultrathin films in Figure 3 as a function of contact separation for a wide range of $t_{\mathrm{YIG}}$ including results on ultrathin YIG films for $400 \mathrm{~nm}$ wide $\mathrm{Pt}$ 
strips and for thicker films $t_{\mathrm{YIG}} \geq 210 \mathrm{~nm}{ }^{6 / 23}$. Figure 44 emphasizes the dramatic enhancement of $R_{\mathrm{nl}}^{1 \omega}$ for the thinnest films down to $t_{\mathrm{YIG}}=3.7 \mathrm{~nm}$ and fixed $d=2.5 \mu \mathrm{m}$, which can be attributed to the $t_{\mathrm{YIG}}$ dependence of $\sigma_{m}$ because $\lambda_{m}>2.5 \mu \mathrm{m}$ for all thicknesses (see SI Section IV for details). $R_{\mathrm{nl}}^{1 \omega}$ increases with decreasing thickness and saturates for both the thinnest and thickest films.

A finite-element model ${ }^{1}$ can simulate the depth $(z)$ dependence of $\mu_{m}$ when $t_{\mathrm{YIG}}>\lambda_{m}$ (see SI Section I for details). This leads to a limiting $\sigma_{m} \rightarrow 3 \times 10^{4} \mathrm{~S} / \mathrm{m}$ in Figure $4 \mathrm{~b}$ for thicker films, which represents the bulk value. The simulated $R_{\mathrm{nl}}$ values for $d=2.5 \mu \mathrm{m}$ in Figure 4 c have been fitted to $R_{\mathrm{nl}}^{1 \omega}$ in Figure $4 \mathrm{a}$ by conductivities that are strongly enhanced in the regime $t_{\mathrm{YIG}}<\lambda_{m}$. For $t_{\mathrm{YIG}}=3.7 \mathrm{~nm}$, the magnon conductivity $\sigma_{m}=1.6 \times 10^{8} \mathrm{~S} / \mathrm{m}$ is four orders of magnitude larger compared to the bulk value, exceeding the electronic conductivity of pure metals such as copper with $\sigma_{e}=6 \times 10^{7} \mathrm{~S} / \mathrm{m}^{24}$. The observed saturation at $t_{\mathrm{YIG}} \rightarrow 0$ appears to reflect an increased role of surface roughness scattering that we do not model explicitly.

A magnon conductivity that diverges for $t_{\mathrm{YIG}} \rightarrow 0$ like $\sigma_{m} \sim \sigma_{m}^{2 \mathrm{D}} t_{\mathrm{YIG}}^{-1}$ simply suggests twodimensional transport. In Figure 4 , it shows that $\sigma_{m}^{2 \mathrm{D}}$ saturates for $t_{\mathrm{YIG}}<10 \mathrm{~nm}$, i.e. higher 2D subbands do not contribute significantly even though they are still populated (see below). Extrapolation to zero thickness leads to $\sigma_{m}^{2 \mathrm{D}} \approx 1 \mathrm{~S}$. This value at room temperature is comparable to that of the high-mobility two-dimensional electron gas at millikelvin temperatures, which is $\sigma_{\mathrm{e}}^{2 \mathrm{D}} \approx 1.4 \mathrm{~S}$ in GaAs quantum wells ${ }^{4}$.

The magnons propagate in the plane with wave vector $\mathrm{k}$ and form perpendicular standing spin waves (PSSW) in $z$ direction labeled by an integer $n$. The exchange interaction scales like $\sim k^{2}$ 
and dominates the magnon dispersion $\varepsilon_{n k}$ at thermal energies $\left(\approx k_{B} T\right)$ with small magnetodipolar corrections. A magnon with energy $\varepsilon_{n k}=\hbar \gamma D\left(k^{2}+\left(n \pi / t_{\mathrm{YIG}}\right)^{2}\right)$ contributes to the conduction proportional to its thermal occupation $N_{n k}=1 /\left\{\exp \left[\varepsilon_{n k} /\left(k_{B} T\right)\right]-1\right\}$, where $\hbar$ is the reduced Planck constant and $k_{B}$ is the Boltzmann constant. For YIG $\gamma / 2 \pi=28 \mathrm{GHz} / \mathrm{T}$ and the spin wave stiffness ${ }^{25} D=5 \times 10^{-17} \mathrm{Tm}^{2}$. The highest occupied subband $n$ defined as

$$
n=\operatorname{int}\left(\frac{t_{\mathrm{YIG}}}{\pi} \sqrt{\frac{k_{B} T}{\hbar \gamma D}}\right)
$$

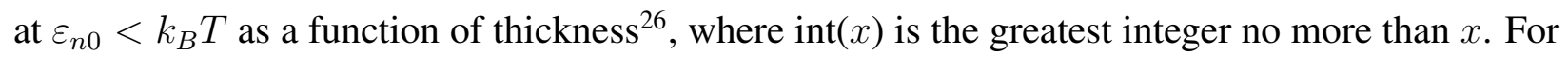
$t_{\mathrm{YIG}}=3.7 \mathrm{~nm}$, only three approximately $2 \mathrm{D}$ subbands are occupied at room temperature.

The simplest model for the magnon conductivity in $\nu(=2,3)$ dimensions follows from the Boltzmann equation with a constant relaxation time $\tau$

$$
\sigma_{m}^{(\nu)}=\frac{e^{2} \tau^{(\nu)}}{k_{B} T} \int \frac{d \mathbf{k}}{(2 \pi)^{\nu}}\left(\frac{\partial \varepsilon_{k}}{\hbar \partial k_{z}}\right)^{2} \frac{e^{\varepsilon_{k} /\left(k_{B} T\right)}}{\left(e^{\varepsilon_{k} /\left(k_{B} T\right)}-1\right)^{2}}
$$

where $\varepsilon_{k}=\hbar \gamma D k^{2}$. Magnetic freeze-out experiments show that the contributions from the lowfrequency magnons $(\sim \mathrm{GHz})$ is significant even at room temperature, presumably reflecting low mobilities of thermal exchange magnons $27-29$. This can be represented by a high momentum cutoff $K_{\infty} \sim 1 / \mathrm{nm}$ at magnon frequencies $\varepsilon_{\infty} / \hbar \sim \mathrm{THz}$. In the high temperature limit $k_{B} T \gg \varepsilon_{k}$ the conductivities do not depend on $\gamma D$ :

$$
\begin{gathered}
\sigma_{m}^{(3)}=\frac{2 e^{2} k_{B} T \tau^{(3)}}{3 \hbar^{2} \pi^{2}} K_{\infty}, \\
\sigma_{m}^{(2)}=\frac{e^{2} k_{B} T \tau^{(2)}}{\pi \hbar^{2}} \log \frac{K_{\infty}}{K_{0}},
\end{gathered}
$$

where $K_{0}$ is a low momentum cutoff by the magnon gap of $\varepsilon_{0} / \hbar \sim \mathrm{GHz}$. By equating these equations with the experimental results $\sigma_{m}^{(3)} \approx 3 \times 10^{4} \mathrm{~S} / \mathrm{m}$ and the present $\sigma_{m}^{(2)} \approx 1 \mathrm{~S}$ and using 
the scattering times as adjustable parameters, we arrive at $\tau^{(3)} \approx 40 \mathrm{fs}$ and $\tau^{(2)} \approx 0.1 \mathrm{~ns}$. The short scattering time in three dimensions can be explained by highly efficient magnon-phonon scattering at room temperature ${ }^{1}$. While the high-momentum cut-off plays an important role in 3 dimensions, the near independence of $\sigma_{m}^{(2)}$ emphasizes the importance of the near band-gap excitations for transport in two dimensions. Coherent magnons excited at $\mathrm{GHz}$ frequencies can propagate over cm's in spite of their small group velocity because they scatter only weakly at phonons ${ }^{30}$. Their contribution has a much larger effect on transport in ultrathin films than in the bulk, which is consistent with the magnetic field and temperature dependence reported in the SI. The estimated scattering time of $\tau^{(2)}=0.1 \mathrm{~ns}$ may be limited by the film roughness scattering. The precise mechanism can be elucidated only by more extensive experimental and theoretical studies of the temperature and field dependence.

While magnon-based devices do not suffer from Joule heating, magnon transport is not dissipationless $\frac{611}{31}$ even for transport on length scales shorter than the magnon relaxation length where magnons are conserved. The observed giant magnon conductivity is therefore excellent news, implying low dissipation from magnon-phonon scattering even at room temperature. Ultrathin films can therefore be driven with relative ease into the non-linear regime in e.g. magnon spin transistors ${ }^{1415}$, facilitating electrically-induced magnon Bose-Einstein condensation and magnon spin superfluidity $32+34$. The robustness of the transport of the magnetic order for thin films of close to the monolayer thickness should allow magnon transport in nanostructures such as constrictions, wires and dots with feature sizes of a few nanometer without loss of magnetic functionality. 


\section{Methods}

Fabrication The YIG films are grown on $\mathrm{Gd}_{3} \mathrm{Ga}_{5} \mathrm{O}_{12}$ (GGG) substrates by liquid-phase epitaxy (LPE) at the Universite de Bretagne Occidentale in Brest, France, with thicknesses from $3.7 \mathrm{~nm}$ to $53 \mathrm{~nm}$ The effective magnetization $M_{\text {eff }}\left(H_{\mathrm{k}}-4 \pi M_{\mathrm{s}}\right)$ and the magnetic relaxation (intrinsic damping parameter $\alpha$ and extrinsic inhomogenous linewidth $\Delta H_{\mathrm{in}}$ ) are determined by broadband ferromagnetic resonance (FMR) in the frequency range 2-40 GHz (see SI Section IV). The device patterns are written by three e-beam lithography steps, each followed by a standard deposition and lift-off procedure. The first step produces a Ti/Au marker pattern, used to align the subsequent steps. The second step defines the platinum injector and detector strips, as deposited by dc sputtering in an Ar+ plasma at an argon pressure with thickness $~ 8 \mathrm{~nm}$ for all devices. The third step defines 5/75 nm Ti/Au leads and bonding pads, deposited by e-beam evaporation. Devices have an injector/detector length $L=30 / 25 \mu \mathrm{m}$ and the strip widths $W$ are $400 \mathrm{~nm}$ for series A and $100 \mathrm{~nm}$ for series B. The experimental results in main text are obtained from series A. The distance-dependent non-local resistances for series B can be found in SI Section III.

Measurements All measurements were carried out by means of three SR830 lock-in amplifiers using excitation frequency of $18 \mathrm{~Hz}$. The lock-in amplifiers are set up to measure the first and second harmonic responses of the sample. Current was sent to the sample using a custom built current source, galvanically isolated from the rest of the measurement equipment. Voltage measurements were made using a custom-built pre-amplifier (gain $10^{3}$ ) and amplified further using the lock-in systems. The typical excitation currents applied to the samples are $200 \mu \mathrm{A}$ (RMS) for series A and 
$20 \mu \mathrm{m}$ for series B. The in-plane coercive field of the YIG $B_{c}$ is below $10 \mathrm{mT}$ for all YIG samples, and we apply an external field to orient the magnetization using a physical property measurement system (PPMS). The samples are mounted on a rotatable sample holder with stepper motor. All experimental data in the main text have been collected at $300 \mathrm{~K}$ (room temperature) at an applied magnetic field of $50 \mathrm{mT}$.

Simulations Our finite-element model implements the magnon diffusion equation in insulators in order to simulate transport of electrically injected magnons. We carried out the simulations by COMSOL MULTIPHYSICS (version 5.4) software package with technical details in the SI Section I.

\section{Acknowledgements}

We acknowledge the helpful discussion with J. Shan and T. Yu. We acknowledge the technical support from J. G. Holstein, H. de Vries, H. Adema, T. Schouten and A. Joshua. This work is part of the research programme "Skyrmionics" with project number 170, which is financed by the Dutch Research Council (NWO). The support by NanoLab NL and the Spinoza Prize awarded in 2016 to B. J. van Wees by NWO is also gratefully acknowledged. G.B. was supported by JSPS Kakenhi Grant 19H00645. 


\section{Author contributions}

B.J.v.W. and X.W. conceived the experiments. X.W. designed and carried out the experiments, with help from O.A.S. J.B.Y. supplied the YIG samples used in the fabrication of devices. X.W., O.A.S., C.H.S.L., G.E.W.B. and B.J.v.W. were involved in the analysis. X.W. wrote the paper with O.A.S., G.E.W.B. and B.J.v.W. All authors commented on the manuscript.

\section{References}

1. Cornelissen, L. J., Peters, K. J. H., Bauer, G. E. W., Duine, R. A. \& van Wees, B. J. Magnon spin transport driven by the magnon chemical potential in a magnetic insulator. Phys. Rev. B 94, 014412 (2016).

2. Chunhui, D. et al. Control and local measurement of the spin chemical potential in a magnetic insulator. Science 357, 195-198 (2017).

3. Olsson, K. S. et al. Pure spin current and magnon chemical potential in a nonequilibrium magnetic insulator. Phys. Rev. X 10, 021029 (2020).

4. Chung, Y. J. et al. Ultra-high-quality two-dimensional electron systems. Nature Materials 20, 632-637 (2021).

5. Brataas, A., van Wees, B., Klein, O., de Loubens, G. \& Viret, M. Spin insulatronics. Physics Reports 885, 1-27 (2020). 
6. Cornelissen, L. J., Liu, J., Duine, R. A., Ben Youssef, J. \& van Wees, B. J. Long-distance transport of magnon spin information in a magnetic insulator at room temperature. Nature Physics 11, 1022-1026 (2015).

7. Lebrun, R. et al. Tunable long-distance spin transport in a crystalline antiferromagnetic iron oxide. Nature 561, 222-225 (2018).

8. Chumak, A. V., Serga, A. A. \& Hillebrands, B. Magnon transistor for all-magnon data processing. Nature Communications 5, 4700 (2014).

9. Cornelissen, L. J., Liu, J., van Wees, B. J. \& Duine, R. A. Spin-current-controlled modulation of the magnon spin conductance in a three-terminal magnon transistor. Phys. Rev. Lett. 120, $097702(2018)$.

10. Barman, A. et al. The 2021 Magnonics Roadmap. Journal of Physics: Condensed Matter 33, 413001 (2021).

11. Chumak, A. et al. Roadmap on Spin-Wave Computing. Preprint at: https://arxiv.org/abs/2111.00365 (2021).

12. Wang, Q. et al. A magnonic directional coupler for integrated magnonic half-adders. Nature Electronics 3, 765-774 (2020).

13. Althammer, M. All-electrical magnon transport experiments in magnetically ordered insulators. physica status solidi (RRL)-Rapid Research Letters 15, 2100130 (2021). 
14. Wimmer, T. et al. Spin transport in a magnetic insulator with zero effective damping. Phys. Rev. Lett. 123, 257201 (2019).

15. Liu, J., Wei, X.-Y., Bauer, G. E. W., Youssef, J. B. \& van Wees, B. J. Electrically induced strong modulation of magnon transport in ultrathin magnetic insulator films. Phys. Rev. B 103, $214425(2021)$.

16. Yu, T., Sharma, S., Blanter, Y. M. \& Bauer, G. E. W. Surface dynamics of rough magnetic films. Phys. Rev. B 99, 174402 (2019).

17. Cherepanov, V., Kolokolov, I. \& L'vov, V. The saga of YIG: Spectra, thermodynamics, interaction and relaxation of magnons in a complex magnet. Physics Reports 229, 81-144 (1993).

18. Li, Y. et al. Hybrid magnonics: Physics, circuits, and applications for coherent information processing. Journal of Applied Physics 128, 130902 (2020).

19. Sinova, J., Valenzuela, S. O., Wunderlich, J., Back, C. H. \& Jungwirth, T. Spin Hall effects. Rev. Mod. Phys. 87, 1213-1260 (2015).

20. Takahashi, S. \& Maekawa, S. Spin injection and detection in magnetic nanostructures. Phys. Rev. B 67, 052409 (2003).

21. Uchida, K. et al. Observation of the spin Seebeck effect. Nature 455, 778-781 (2008).

22. Gomez-Perez, J. M., Vélez, S., Hueso, L. E. \& Casanova, F. Differences in the magnon diffusion length for electrically and thermally driven magnon currents in $\mathrm{Y}_{3} \mathrm{Fe}_{5} \mathrm{O}_{12}$. Phys Rev. B 101, 184420 (2020). 
23. Shan, J. et al. Influence of yttrium iron garnet thickness and heater opacity on the nonlocal transport of electrically and thermally excited magnons. Phys. Rev. B 94, 174437 (2016).

24. Laughton, M. A. \& Say, M. G. Electrical engineer's reference book (Elsevier, 2013).

25. Klingler, S. et al. Measurements of the exchange stiffness of YIG films using broadband ferromagnetic resonance techniques. Journal of Physics D: Applied Physics 48, 015001 (2014).

26. Stamps, R. \& Camley, R. Solid State Physics. No. Volume 65 in Solid State Physics (Elsevier Science, 2014).

27. Kikkawa, T. et al. Critical suppression of spin Seebeck effect by magnetic fields. Phys. Rev. $B$ 92, 064413 (2015).

28. Jin, H., Boona, S. R., Yang, Z., Myers, R. C. \& Heremans, J. P. Effect of the magnon dispersion on the longitudinal spin Seebeck effect in yttrium iron garnets. Phys. Rev. B 92, 054436 (2015).

29. Jamison, J. S. et al. Long lifetime of thermally excited magnons in bulk yttrium iron garnet. Phys. Rev. B 100, 134402 (2019).

30. Streib, S., Vidal-Silva, N., Shen, K. \& Bauer, G. E. W. Magnon-phonon interactions in magnetic insulators. Phys. Rev. B 99, 184442 (2019).

31. Man, H. et al. Direct observation of magnon-phonon coupling in yttrium iron garnet. Phys. Rev. B 96, 100406 (2017).

32. Bender, S. A., Duine, R. A. \& Tserkovnyak, Y. Electronic Pumping of Quasiequilibrium Bose-Einstein-Condensed Magnons. Phys. Rev. Lett. 108, 246601 (2012). 
33. Demokritov, S. O. et al. Bose-Einstein condensation of quasi-equilibrium magnons at room temperature under pumping. Nature $\mathbf{4 4 3}, 430-433$ (2006).

34. Divinskiy, B. et al. Evidence for spin current driven Bose-Einstein condensation of magnons. Nature Communications 12, 6541 (2021). 
a.

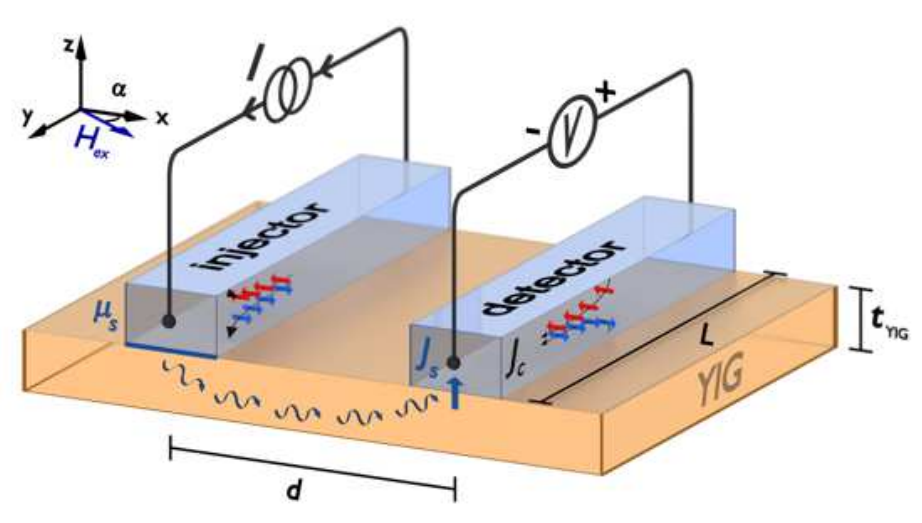

b.

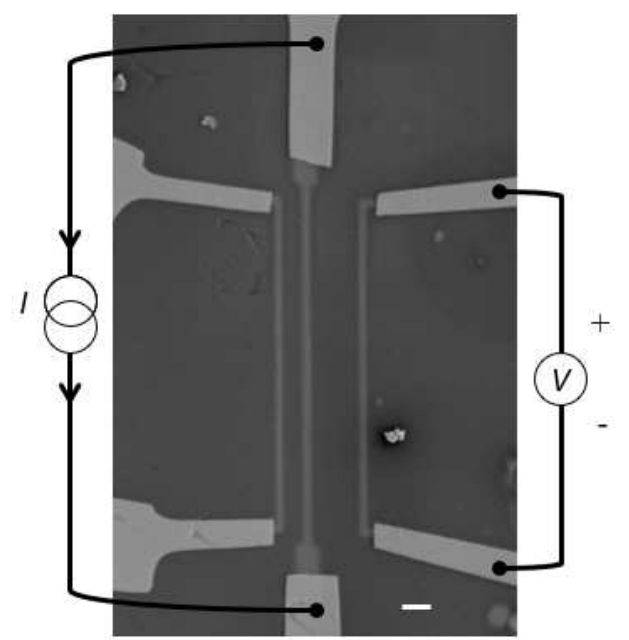

Figure 1: Device layout: a) Schematic representation of the experimental geometry. Two Pt strips deposited on top of YIG serve as magnon detector and injector via the direct and inverse spin hall effect. A low-frequency ac current with rms value of $I_{\mathrm{ac}}$ through the left Pt strip injects magnons. The center-to-center distance of the injector and the detector is $d$ and the length of the injector/detector is $L$. A spin accumulation $\mu_{s}$ is formed at Pt|YIG interface due to the SHE when a charge current passes through the injector and excites magnon non-equilibrium underneath the injector. The diffusive magnons are absorbed at the drain, which induce a spin current density $j_{s}$. Using a lock-in technique, the first harmonic voltage is measured simultaneously by the right Pt strip, i.e. a magnon detector. $\alpha$ is the angle of external magnetic field $H_{\text {ex }}$. b) SEM image of the geometry. The parallel vertical lines are the platinum injector and detector, and they are contacted by gold leads. Current and voltage connections are indicated schematically. The scale bar represents $2 \mu \mathrm{m}$. 


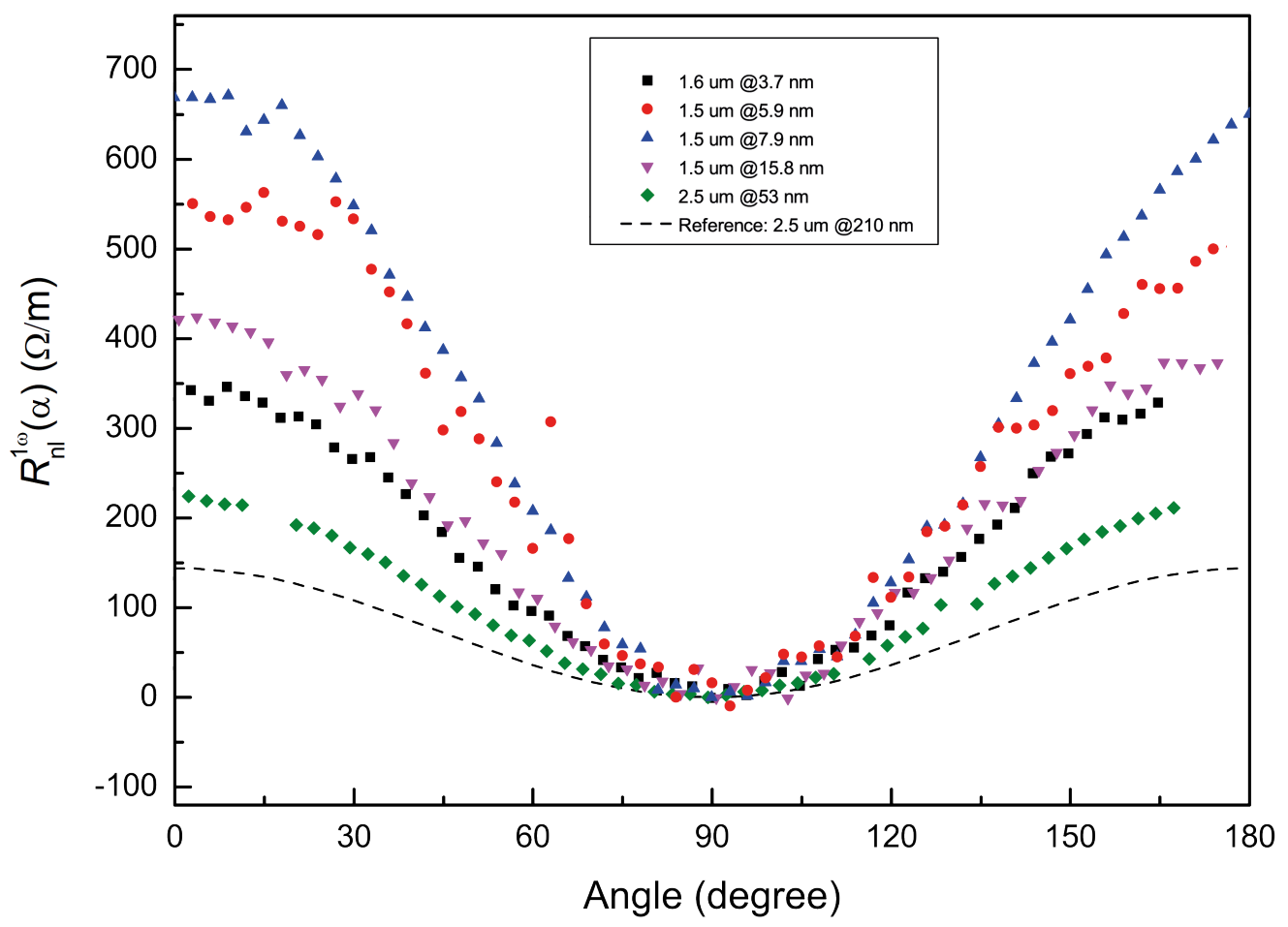

Figure 2: Dependence of non-local resistance on magnetization direction on ultrathin YIG films, for short center-to-center distances between the injector and the detector. The offset $R_{0}^{1 \omega}$ in Eq 4 has been subtracted. This shows $R_{\mathrm{nl}}^{1 \omega}$ increases with decreasing thickness. Comparing with the reference from Cornelissen et al.,$R_{\mathrm{nl}}^{1 \omega}$ significantly increases in ultrathin films. 


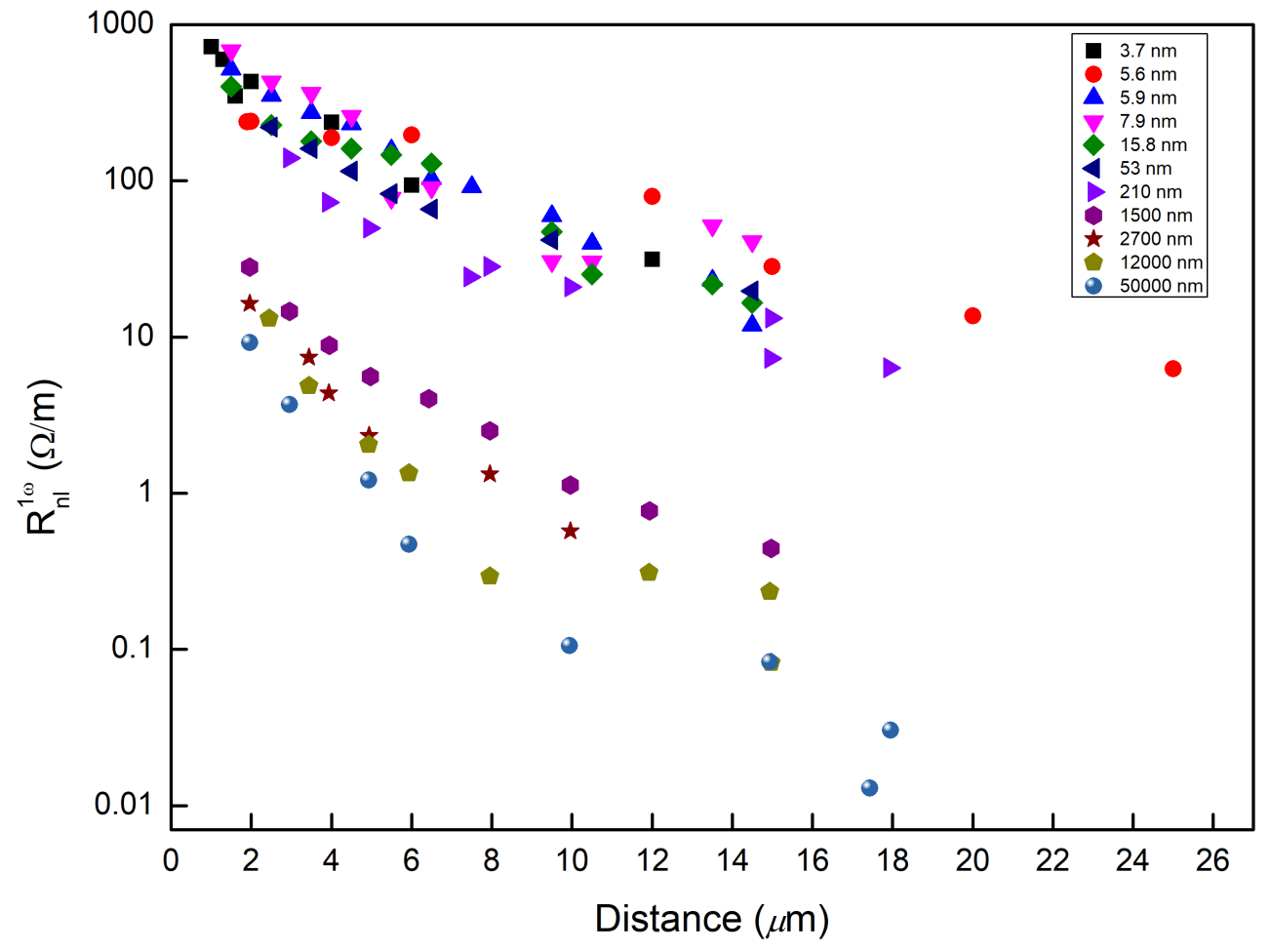

Figure 3: Non-local resistance as a function of injector-detector distance of the samples of series A and $t_{\text {YIG }}$ varying from $3.7 \mathrm{~nm}$ to $50000 \mathrm{~nm}$. The width of injector/detector is $400 \mathrm{~nm}$. The results for $t_{\mathrm{YIG}} \geq 210 \mathrm{~nm}$ are adopted from Cornelissen et al. ${ }^{[6}$ and Shan et al. ${ }^{23}$. 

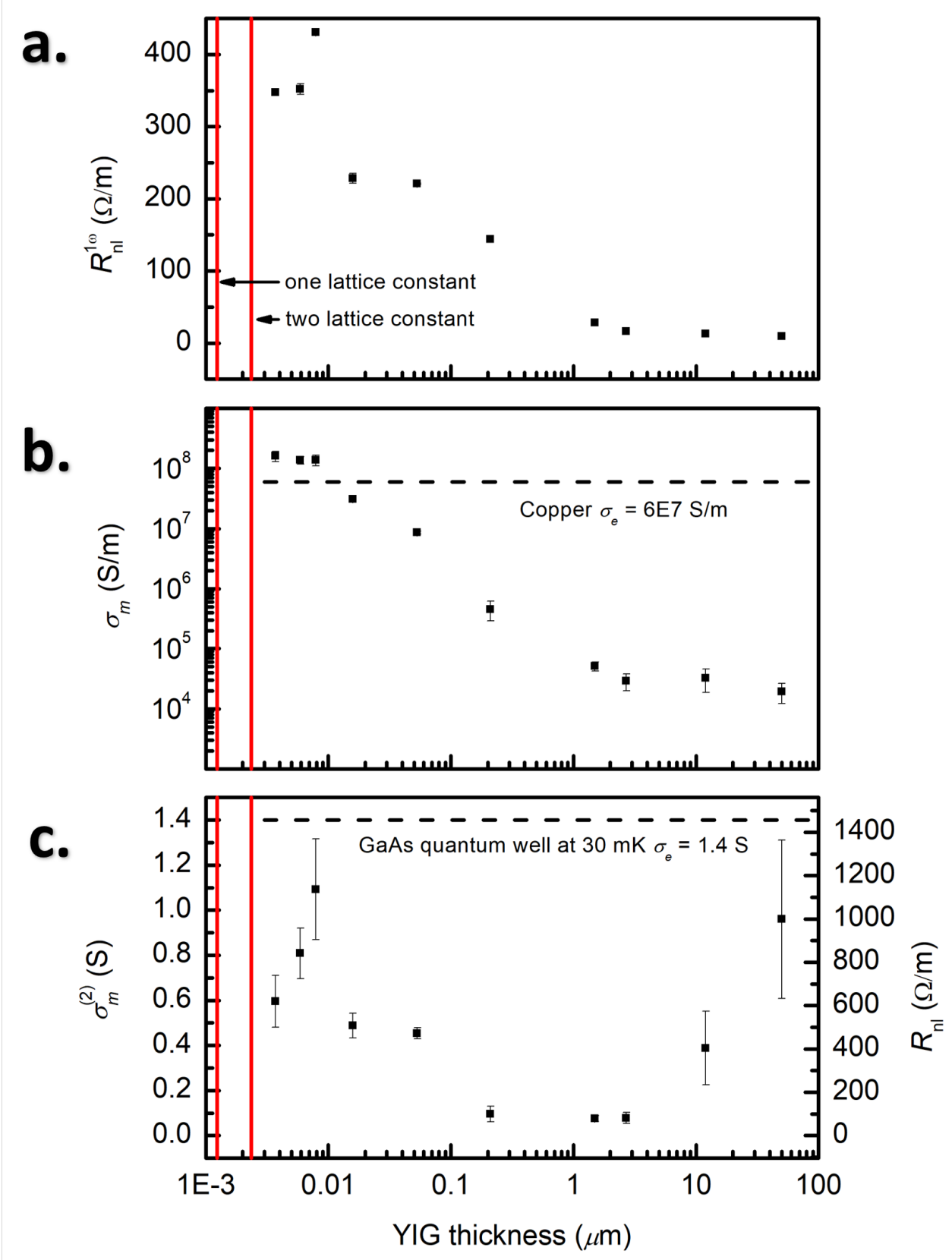

Figure 4: a) The non-local resistance $R_{\mathrm{nl}}^{1 \omega}$ at $d=2.5 \mu \mathrm{m}$ as a function of $t_{\mathrm{YIG}}$. The results for $t_{\text {YIG }} \geq 210 \mathrm{~nm}$ are adopted from Cornelissen et al. ${ }^{[6]}$ and Shan et al. ${ }^{[23}$. b) Thickness dependence of the magnon conductivity $\sigma_{m}$ obtained by the best fit for different distances with statistical error bars. c) Thickness dependence of the 2-dimension spin conductance $\sigma_{m}^{(2)}$ and the non-local resistance $R_{\mathrm{nl}}$ from the simulation, values based on the best fit for the magnon conductivity. The saturation at $t_{Y I G} \rightarrow 0$ indicates that the film approaches the two-dimensional regime in ultrathin limit. The obtained error bar here means the range of the best fitting results for the non-local resistance we can get from the FEM simulations (see SI Section II). 


\section{Supplementary Files}

This is a list of supplementary files associated with this preprint. Click to download.

- Supplementary.pdf 